\title{
Tuning the Magnetic Properties of Diamagnetic Di-Blatter's Zwitterion to Antiferro- and Ferromagnetic Diradical
}

\author{
Rishu Khurana, ${ }^{\dagger, \ddagger}$ Ashima Bajaj, ${ }^{\dagger, \ddagger}$ and Md. Ehesan Ali; ${ }^{* \dagger}$ \\ $\dagger$ Institute of Nano Science and Technology, Sector-81, Mohali, Punjab-140306, India \\ $\ddagger$ These authors contributed equally to this work. \\ E-mail: ehesan.ali@inst.ac.in
}

\begin{abstract}
In the quest of obtaining organic molecular magnets based on stable diradicals, we have tuned the inherent zwitterionic ground state of tetraphenylhexaazaanthracene (TPHA), the molecule embraced with two Blatter's moieties, by adopting two different strategies. In the first strategy, we have increased the length of the coupler between the two radical moieties and observed a transition from zwitterionic ground state to diradicalized state. With larger coupler, remarkably strong ferromagnetic interactions are realized based on DFT and WFT based CASSCF/NEVPT2 methods. An analysis based on extent of spin contamination, CASSCF orbitals occupation numbers, HOMOLUMO and SOMOs energy gap is demonstrated that marks the transition of ground state in these systems. In another approach, we systematically explore the effect of push-pull substitution on the way to obtain molecules based on TPHA skeleton with diradicaloid state and in some cases, even triplet ground state.
\end{abstract}




\section{Introduction}

Stable organic radicals have become the appealing candidates due to their peculiar electronic and magnetic properties. ${ }^{1}$ Owing to their fascinating applications in organic spintronics, ${ }^{2,3}$ energy storage devices, ${ }^{4}$ organic field effect transistors etc, ${ }^{5,6}$ delocalized diradicals have gained utmost importance. Hence, it becomes the necessity to understand spin-spin interactions and the nature of chemical bond between them. ${ }^{7-9}$ They can be classified into Kekulé and non-Kekulé molecules. ${ }^{10}$ In Kekulé structures, there exist a "resonance hybrid" between open shell and closed shell configurations. ${ }^{11}$ In the open shell diradical configuration, the aromatic six-membered rings are formed, which can vanquish the energy for the breaking of a carbon-carbon $\pi$-bond. Kekulé molecules (diradicaloids) have been substantially explored as they exhibit low band gap energy and convertible spin state. ${ }^{12,13}$ However, in non-Kekulé structures, some electrons are unpaired due to the topology of the $\pi$-electron arrangement. ${ }^{14}$

The singlet diradicaloids, that is, the molecules with partial singlet diradical nature in their ground state are expected to have unique structure with interesting electronic, optical and magnetic properties. But the major challenging part is their structural instability and synthetic difficulties. However, after the pioneering reports of carbon-centered diradicaloids, namely Thiele's hydrocarbons in $1904^{15}$ and Tschitschibabin's hydrocarbon in $1907,{ }^{16}$ the research on this class of compounds has been unrolled widely. A series of diradicaloids which includes bisphenalyenyls ${ }^{17,18}$, quinodimethanes ${ }^{19}$, diiendenoacenes ${ }^{20}$, indenofluorenes ${ }^{21}$, zenthrenes $^{22}$ and anthenes ${ }^{23}$ etc. have been synthesized. Following this, a number of diradicals based on stable known radicals like nitronyl nitroxide, imino nitroxide, verdazyl, oxoverdazyl etc. have been synthesized enormously. ${ }^{24-33}$ Another super-stable monoradical is 1,3-diphenyl-1,2,4-benzotriazinyl (Blatter's) radical reported by Blatter and co-workers in 1968. ${ }^{34}$ However, the radical gained popularity only after the pioneering efforts by Koutentis and his group for its easy synthetic strategies. ${ }^{35-37}$ Several studies on the synthesis, applica-

tions and magnetic properties of the Blatter's radicals have been done so far. ${ }^{38-44}$ Rajca et 
al. synthesized the stable diradicals by coupling the Blatter's radical with nitronyl nitroxide and imino nitroxide with superior magnetic properties. ${ }^{45,46}$ Subsequently, we proposed several hybrid diradicals by coupling Blatter's radical with other stable radical moieties including nitronyl nitroxide, imino nitroxide, oxoverdazyl, dithiadiazolyl, phenoxy and nitroxy as spin sources and observed strong ferromagnetic exchange interactions employing state-ofart multireference (CASSCF-NEVPT2) methods. ${ }^{47}$ Later on, we witnessed an appreciable enhancement in ferromagnetic exchange couplings by utilizing oxygen-functionalized planar analogues of Blatter's radical. ${ }^{48}$ The superior air and moisture stability and thermally robust properties of Blatter's radical also led Wudl and group to synthesize stable benzotriazinyl diradicals. ${ }^{49,50}$ Thereafter, Zheng et al. proposed the synthesis of Blatter's diradicals by coupling the two monomers of Blatter's radical. ${ }^{51,52}$ Prompted by this, we investigated different possibilities of coupling the two Blatter's radical via its fused benzene ring and realized that it is quite challenging to obtain di-Blatter diradicals with ferromagnetic interactions due to several micro-magnetic exchange interactions existing between the localized radical centers. ${ }^{53}$ A stable star-shaped triradical implementing Blatter's radical has been recently synthesized by Zissimou et al. ${ }^{54}$

Tetraphenylhexaazaanthracene (TPHA), the earliest compound containing Blatter's radical moiety, was synthesized by Hutchison et al. with the possibility of obtaining stable heteroatomic ferromagnetic diradical with m-phenylene as coupler. ${ }^{55}$ However, contrary to the expectations, it was found to exist as exceptionally stable zwitterionic system with a closed shell ground-state possessing diamagnetic behaviour that undergoes photo-induced intramolecular electron transfer to form a radical pair (Figure 1). This molecule is known to exhibit large singlet-triplet energy difference $\left(\Delta \mathrm{E}_{C S S-T}\right)$ of $-20.1 \mathrm{kcal} / \mathrm{mol}$ and overcome its $16 \pi$ potential antiaromaticity by sacrificing the aromaticity of the central fused benzene ring and partitioning itself into $10 \pi$-anionic and $6 \pi$-cationic sub-units which are structurally bridged by $\sigma$-bonds but are not conjugated electronically. Further, efforts have been made to reduce this veritable energy barrier $\left(\Delta \mathrm{E}_{C S S-T}\right)$ but the triplet ground state has not been 
achieved so far. ${ }^{56}$ The two analogous isomers of TPHA possessing closed-shell ground state have been isolated and identified as minor side products by Constantinides et al. ${ }^{57}$ Haas et al. rationalised the existence of these zwitterions due to electron transfer from donor to acceptor sub-units. The transfer is possible only if the donor has low ionization potential and the acceptor has high electron affinity. ${ }^{58}$ Further, another molecule, tetraphenylhexaazaacridine (TPH-acridine) was synthesized by Langer et al. which was observed to be weakly antiaromatic and highly zwitterionic similar to TPHA. ${ }^{59}$ Braunstein et al. also observed the partitioning of $12 \pi$ electrons into two $6 \pi$ sub-units which are not electronically conjugated to form zwitterionic benzoquinonemonoimine. ${ }^{60}$

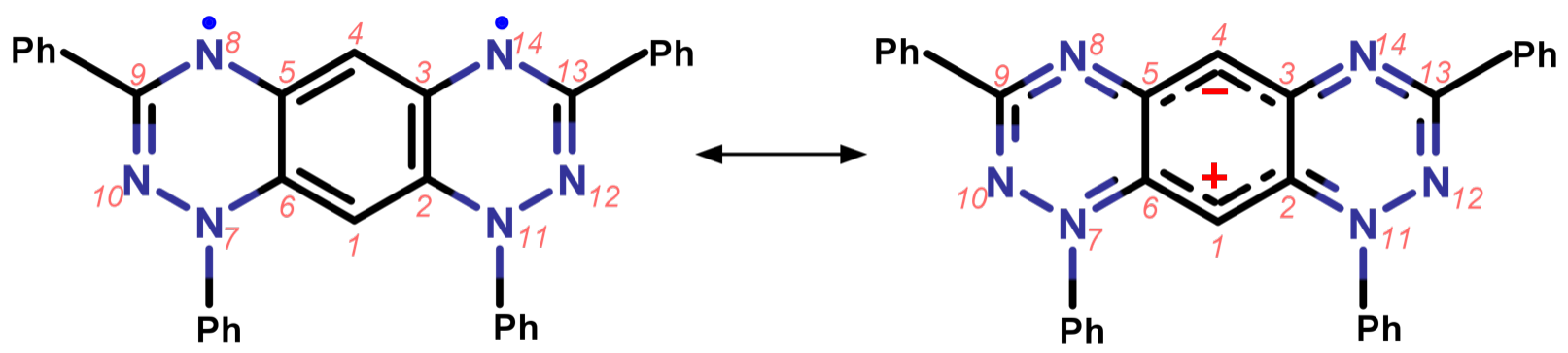

Figure 1: Resonating structures of TPHA indicating resonance between the photoexcited diradical and the zwitterionic ground state forms.

Since, polyacenes are known to show inherent property of increase in the diradical character with the increase in the number of fused benzene rings i.e. their length, which can facilitate the enhanced spin-spin coupling between the radical centers. ${ }^{61,62}$ Taking advantage of this peculiar property, the quest arises, can we manipulate the well known zwitterionic ground state of TPHA by utilizing polyacene as a coupler to accomplish strong ferromagnetic exchange interactions? Here, we demonstrated an exclusive transition from the zwitterionic closed shell ground state of TPHA to ferromagnetically coupled Blatter's diradicals by modifying the length of the coupler. Several factors including diradical character, extent of spin contamination, alignment of frontier orbitals etc, have been used as guiding principles to mark this spin-state transition with the increasing length of coupler. Apart from this, many experimental and theoretical studies have reported the molecules with zwitterionic ground 
state where the introduction of EDG and EWG can influence the diradical character and hence, their ground state. ${ }^{63-65}$ However, the effect of simultaneous substitution of EDG and EWG i.e. push-pull substitution has not been studied so far. Therefore, in this work, we have also explored the effect of push-pull substitution with the replacement of hydrogen atoms of fused benzene ring of TPHA skeleton. Thus, approaching towards the development of molecules with diradicaloid ground state.

\section{Theoretical Methods and Computational Details}

The magnetic exchange interaction $(2 J)$ between the two magnetic sites A and B appears in the Heisenberg effective spin Hamiltonian as

$$
\hat{H}=-2 J \hat{S}_{A} \cdot \hat{S_{B}}
$$

where $\hat{S_{A}}$ and $\hat{S_{B}}$ are the spin angular momentum operators on the interacting sites $\mathrm{A}$ and B respectively and $2 J$ is the exchange coupling parameter. With positive $2 J$, high-spin triplet state is lower in energy with ferromagnetic coupling between the two spin carrier sites. A negative $2 J$ represents the low-spin singlet as ground state with antiferromagnetic interactions. For a diradical with two unpaired electrons on each magnetic site, $2 J$ can be represented as the energy difference between the singlet $\left(E_{S}\right)$ and triplet $\left(E_{T}\right)$ spin state i.e.

$$
E_{S}-E_{T}=2 J
$$

In the conventional single-reference methods (e.g UKS, UHF), the singlet state of a diradical cannot be expressed due to multi-determinantal nature of the low spin-state wavefunction. As an alternative approach, the magnetic exchange coupling constant $(2 J)$ can be extracted using the broken-symmetry approach within the DFT framework as proposed by Noodleman 
et al. ${ }^{66}$ expressed as

$$
2 J=\frac{2\left(E_{B S}-E_{T}\right)}{S_{\max }^{2}}
$$

where $\mathrm{E}_{B S}$ and $\mathrm{E}_{T}$ refer to the energies of broken-symmetry and triplet states and $S_{\max }$ represents the total spin of high-spin state. This assumes that BS state is an equal mixture of highest and lowest spin states wavefunction. Further, to study the density of local aromaticity in polyacene molecules, the Harmonic Oscillator Model of Aromaticity (HOMA) index is calculated according to Kygowski et al. ${ }^{67,68}$ as

$$
H O M A=1-\frac{\alpha}{n} \sum_{i=1}^{n}\left(R_{i}-R_{o p t}\right)^{2}
$$

where $\alpha$ is the normalization factor $(257.7), n$ is the number of $\mathrm{C}-\mathrm{C}$ bonds, $\mathrm{R}_{i}$ is the bond length computed from DFT calculations, $\mathrm{R}_{o p t}$ is the optimal bond length for aromatic compounds with value of $1.388 \AA$ for $\mathrm{C}$-C bond. HOMA is defined as the normalized sum of squared deviations of bond lengths from the optimal value that are assumed for a fully aromatic system. For a fully aromatic system, HOMA $=1$ and for non-aromatic systems, $\mathrm{HOMA}=0$.

For all the molecules under study, the geometries were optimized employing the Becke's three-parameter hybrid functional (B3LYP) ${ }^{69,70}$ and atom centred polarized triple-zeta (def2TZVP) basis set ${ }^{71}$ in three distinct electronic states, i.e., broken-symmetry open-shell singlet (OSS) state, open-shell triplet (T) state and closed-shell singlet (CSS) state. OSS and T states are optimized within spin-unrestricted formalism, whereas spin-restricted wavefunction is used for CSS state. Resolution of the identity (RI) approximation in conjunction with auxiliary basis set def2/J and chain of spheres (COSX) numerical integration was used to speed up the calculations. ${ }^{72}$ The exchange interactions were computed applying BS-DFT method employing Eq. 3. ${ }^{73-75}$ Apart from DFT based methods, the magnetic exchange interactions were also calculated employing wave-function based complete active space self consistent field (CASSCF) wherein the $2 J$ values are obtained using Eq. 2. ${ }^{76,77}$ Further, 
the dynamical correlations were included using N-electron valence state perturbation theory (NEVPT2) method. ${ }^{78}$ To quantify the diradical character, the CASSCF $(10,10) /$ def2-TZVP was used to calculate the orbital occupation number of the molecules. All the calculations are performed in ORCA (version 4.0.1.2) quantum chemical code. ${ }^{79}$

\section{Results and Discussion}

\subsection{Increasing the length of coupler}

The two Blatter's radical are coupled to each other through its fused benzene ring, wherein $n=1$ corresponds to TPHA. Afterwards, one benzene unit is consecutively added in the spacer between the two radical centers upto hexaacene $(n=6)$ as shown in Figure 2. All the molecules

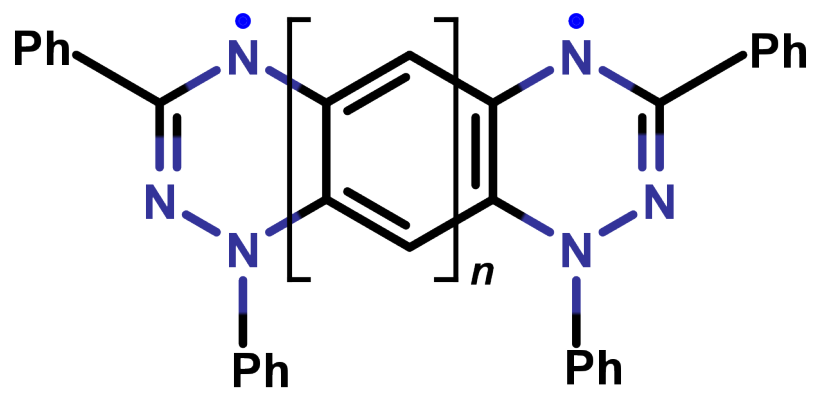

Figure 2: Two Blatter radicals coupled through benzene ring. Here, $n=1$ to 6 , corresponds to number of benzene rings used to couple two radical moieties. The corresponding molecules are aliased as $\mathbf{1}$ to $\mathbf{6}$ depending on the number of benzene rings i.e. $\mathbf{1}$ corresponds to molecule containing 1 benzene ring.

are optimized in the three electronic states, i.e., closed-shell singlet (CSS), broken-symmetry open-shell singlet (OSS) and open-shell triplet (T) states. The DFT optimized energies in all the states are given in Table S1. The relative energies i.e. $\Delta \mathrm{E}_{X-C S S}$ (where $\mathrm{X}=\mathrm{CSS}$, OSS, T) estimated from the relative energy difference between the DFT optimized energies are shown in Figure 3 and are collected in Table S2. It is observed that the molecule $\mathbf{1}$ preferred the closed-shell singlet as ground state as reported earlier. ${ }^{55}$ It is also observed that although 
the calculation was performed in spin-unrestricted (UKS) case, it was found to reduce to spin-restricted (RKS) solution and almost identical energies with a minute difference of 0.8 meV between the OSS and CSS states were obtained. Molecule 2 also exhibits closed shell ground-state with OSS state lying $11.8 \mathrm{meV}$ above the CSS state. However, for molecule 3 and 4 , larger $\triangle \mathrm{E}_{O S S-C S S}$ is observed indicating the instability of the CSS structure i.e. these molecules favour the OSS as the ground state. Further, with larger number of benzene rings i.e. for molecule 5 and $\mathbf{6}$, there is substantial increase in $\Delta \mathrm{E}_{T-C S S}$ representing the preference of triplet as the ground state.

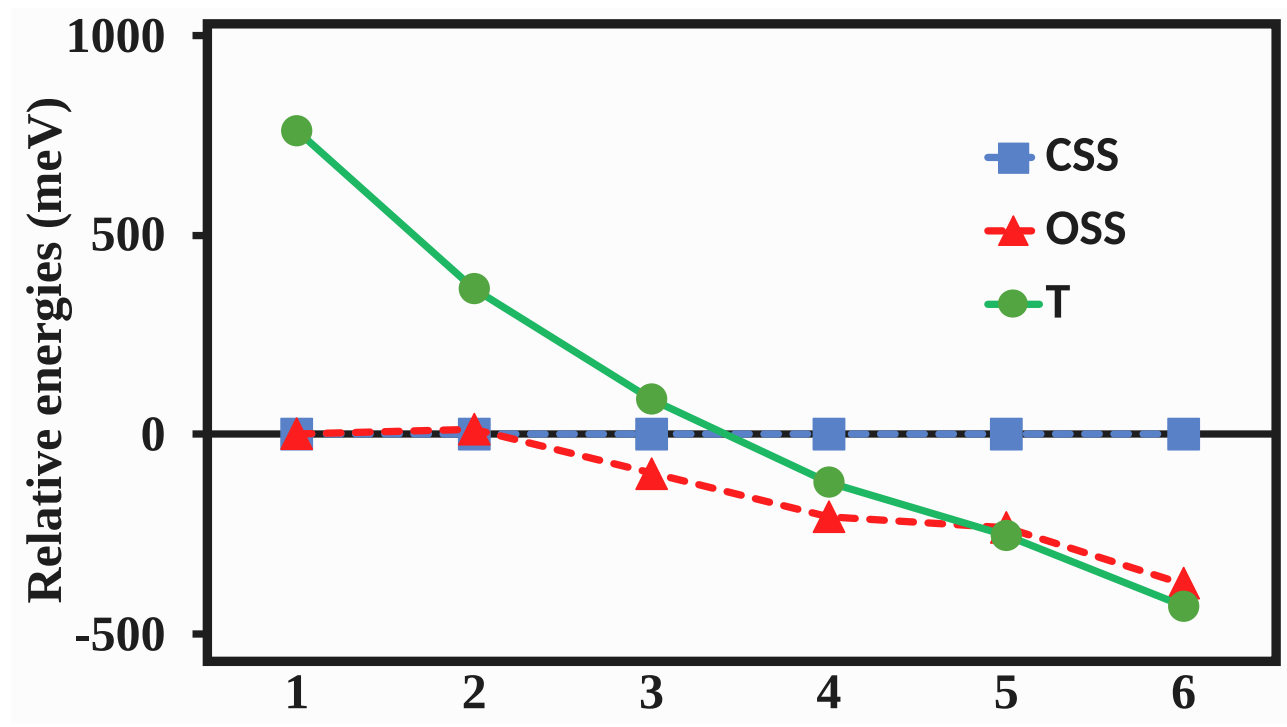

Figure 3: Relative energies (meV) of the CSS, OSS and T states (referred to the CSS) of molecule 1 to 6 .

The spin-squared value i.e. $\left\langle S^{2}>\right.$ has been used to estimate the extent of diradical character in a molecule. The spin contaminations can be determined from the calculated values of $\left\langle S^{2}>\right.$. The $\left\langle S^{2}>\right.$ value should be 2.0 for the triplet-state diradical and 1.0 for the pure singlet-state using the BS method. Any deviation from the ideal $\left\langle S^{2}\right\rangle$ values indicates the spin contamination. From the $\left\langle S^{2}>\right.$ values in HS state (Figure 4 and Table 1), it is observed that the spin contamination for molecules $\mathbf{1}$ to $\mathbf{4}$ are low and the deviations from the expected value of 2.0 is atmost 0.08. However, for molecules $\mathbf{5}$ and $\mathbf{6}$, the spin contamination is observed to be 0.36 . The reason for the increased spin contamination in HS 
state for larger polyacene couplers is the enhanced degenerate nature of molecular orbitals as $n$ increases (Figure S1) which is also observed in one of the author's previous work. ${ }^{80}$

Table 1: Computed spin-squared value $\left(<S^{2}>\right)$ in HS and BS state for all the molecules and calculated magnetic exchange coupling constants for the diradicals 3-6.

\begin{tabular}{cccccc}
\hline Molecule & $<S^{2}>_{H S}<S^{2}>_{B S}$ & \multicolumn{2}{c}{$2 J\left(\mathbf{c m}^{-1}\right)$} \\
& \multicolumn{2}{c}{ BS-DFT } & BS-DFT & CAS(10,10) & CAS(10,10)/NEVPT2 \\
\hline $\mathbf{1}^{a}$ & 2.03 & 0.23 & - & - & - \\
$\mathbf{2}^{a}$ & 2.03 & 0.56 & - & - & - \\
$\mathbf{3}$ & 2.04 & 0.77 & -2774.64 & -333.08 & -1551.44 \\
$\mathbf{4}$ & 2.08 & 0.89 & -1240.04 & -58.30 & -447.03 \\
$\mathbf{5}$ & 2.21 & 0.97 & 328.84 & 22.38 & 37.10 \\
$\mathbf{6}$ & 2.36 & 1.03 & 1797.40 & 823.03 & 950.10 \\
\hline
\end{tabular}

${ }^{a}$ These molecules possess zwitterionic ground state that behave as diamagnetic species with closed-shell singlet(CSS) ground state with large $\Delta \mathrm{E}_{S T}\left(>5000 \mathrm{~cm}^{-1}\right)$.

However, as illustrated in Figure 4 and Table 1, the computed $<S^{2}>$ values in BS state are found to be significantly less than 1.0. Thus, indicating the pronounced contribution of closed shell singlet electronic configuration in the singlet diradicals. A large deviation of $<S^{2}>$ values in BS state from the expected value of 1.0 is observed for molecule $\mathbf{1}(0.23)$, clearly indicating the existence of the molecule in the CSS ground state which agrees well with the predicted state of this molecule. However, as the number of benzene rings i.e. $n$ increases between the two spin centered Blatter's radicals, the value starts approaching towards 1.0 indicating the preferential existence of molecule in the diradical configurations. For molecules 3 and $\mathbf{4}$, the $\left\langle S^{2}>\right.$ value is 0.77 and 0.89 which means that they are indeed diradicalized and their ground state is open shell singlet, however, their degree of diradical character is not as ideal as for pure singlet-state (BS). However, for $\mathbf{5}$ and $\mathbf{6},<S^{2}>$ is $\sim 1.0$, indicating the attainment of ideal diradical state in these molecules. These observations are in line with those observed with the relative energies discussed above. 


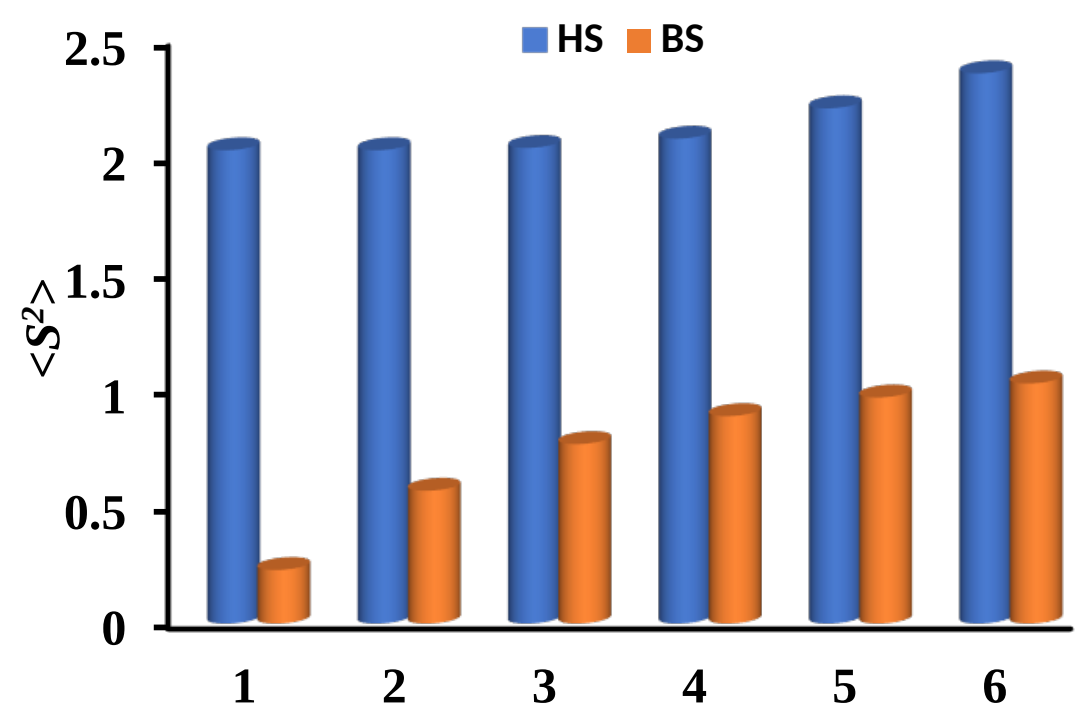

Figure 4: Computed $<S^{2}>$ values in HS and BS state for the molecules 1 to 6 obtained at UB3LYP/def2-TZVP level.

Another index for the evaluation of diradical character in a molecule is the occupation number of lowest unoccupied natural orbital $\left(n_{L U N O}\right)$ obtained from the CASSCF calculations. The percentage of diradical character can be directly estimated from $n_{L U N O}$ by simply multiplying it with 100. Therefore, we calculated the occupation number of highest occupied natural orbital (HONO) and lowest unoccupied natural orbital (LUNO) and the percentage of diradical character using the $\operatorname{CASSCF}(10,10) /$ def2-TZVP method. For molecule $\mathbf{1}$, the LUNO occupation number, $n_{L U N O}$, is observed to be 0.30 indicating that it has only $30 \%$ diradical character which further confirms the zwitterionic character of the molecule. As we proceed towards molecule with increasing number of benzene rings, the occupation number of LUNO goes on increasing. It is observed to be $0.58,0.80,0.89,0.94$ and 0.95 for molecule $\mathbf{2}, \mathbf{3}, \mathbf{4}, \mathbf{5}$ and $\mathbf{6}$ respectively inferring $58 \%, 80 \%, 89 \%, 94 \%$ and $95 \%$ diradical character in these molecules (Table S3). The significant increase in diradical character from $30 \%$ for $\mathbf{1}$ to $95 \%$ for $\mathbf{6}$ unambiguously confirms the evolution of a diradical state with the increasing length of coupler. The diradical indexes derived employing two different calculation methods are found to be in agreement with each other confirming the extent of diradical character in these molecules. 
Further to find out the relationship between the ground-state and the magnetic spin couplings, correlation diagram is plotted between the magnetic exchange coupling constant, $2 J\left(\mathrm{~cm}^{-1}\right)$ and the energy gap between the closed-shell singlet and triplet i.e. $\Delta \mathrm{E}_{C S S-T}$ (kcal/mol) as shown in Figure 5a. The degree of correlation is quantified on the basis of correlation coefficient i.e. $\mathrm{R}^{2}$ which describes the strength of relationship between the two variables. A value of 1.0 and -1.0 indicates strong positive and negative correlations and a value of 0 means there is no correlation between the two variables. A highly linear correlation with correlation coefficient $\left(\mathrm{R}^{2}\right)$ of 0.99 is found between $2 J$ and $\Delta \mathrm{E}_{C S S-T}$. The large negative energy gap and high negative magnetic exchange coupling constant $(2 J)$ are in line with the zwitterionic character of molecules $\mathbf{1}$ and $\mathbf{2}$. As the energy gap decreases and comes in the range of -5 to $5 \mathrm{kcal} / \mathrm{mol}$ i.e. for 3 and 4 , open shell singlet emerges as the ground state with antiferromagnetic exchange interactions between the two radical centers. However, with energy gap $>5 \mathrm{kcal} / \mathrm{mol}$ i.e. for molecule $\mathbf{5}$ and $\mathbf{6}$, ferromagnetic interactions are favoured. The diradical character emerges from the interaction between the singlet and the triplet states. ${ }^{81}$ It is observed that if the energy gap between the closed-shell singlet and triplet is high, the configuration interaction between them is negligible and the molecule has preferential closed-shell ground state. However, if the singlet-triplet energy gap is small, they have strong interactions between them and hence, the molecule prefers the open-shell singlet or triplet as ground state. 


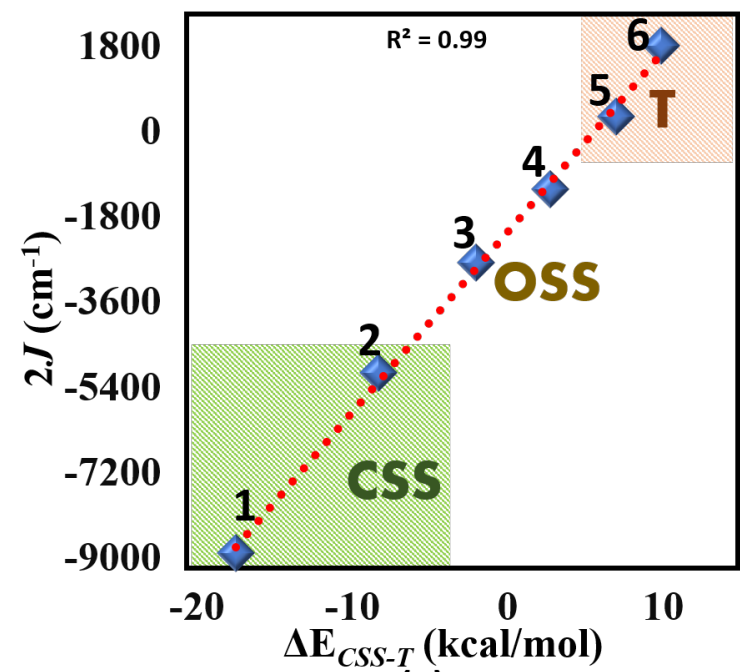

(a)

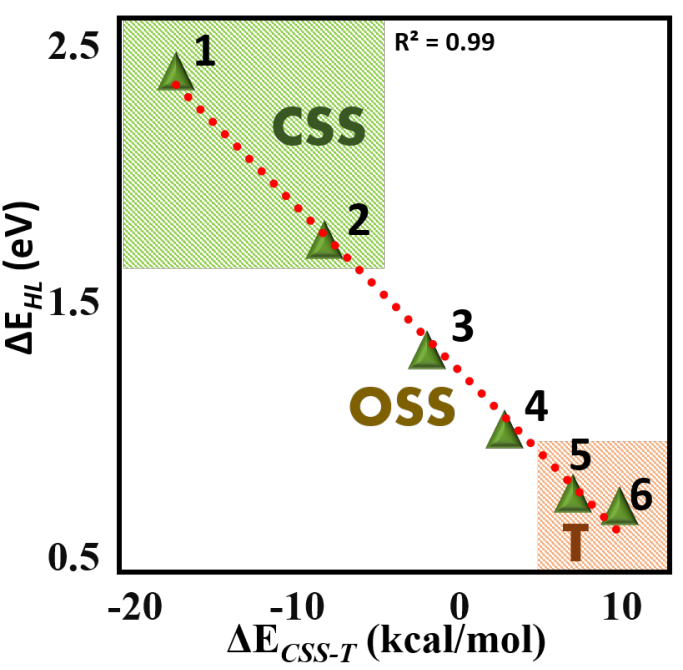

(b)

Figure 5: Linear correlation diagram (a) between the magnetic exchange coupling constant, $2 J\left(\mathrm{~cm}^{-1}\right)$ and the energy gap between the closed-shell singlet and triplet states i.e. $\Delta \mathrm{E}_{C S S-T}$ (kcal/mol) (b) between HOMO-LUMO gap i.e. $\Delta \mathrm{E}_{H L}(\mathrm{eV})$ in the closed-shell state and $\Delta \mathrm{E}_{C S S-T}(\mathrm{kcal} / \mathrm{mol})$ for molecules 1 to $\mathbf{6}$ with correlation coefficient i.e. $\mathrm{R}^{2}$ of 0.99 .

To realize the relationship between singlet-triplet energy gap i.e. $\Delta \mathrm{E}_{C S S-T}$ and HOMOLUMO energy gap i.e. $\Delta \mathrm{E}_{H L}$, we plotted the correlation diagram between the two. The singlet-triplet energy gap is observed to be in close alliance with the HOMO-LUMO gap that is observed from the linear correlation diagram with high correlation coefficient $\left(\mathrm{R}^{2}\right)$ of 0.99 as shown in Figure 5b. Thus, to realize the extent of diradical character in these systems and hence, their ground spin state, it becomes essential to further investigate the HOMO-LUMO gap. The computed HOMO-LUMO gap i.e. $\Delta \mathrm{E}_{H L}$ (in $\mathrm{eV}$ ) is observed to decrease smoothly with increasing the length of the coupler. It is found to be much smaller in the diradicals $\mathbf{3}(1.25), \mathbf{4}(0.94), \mathbf{5}(0.64)$ and $\mathbf{6}(0.39)$ as compared to the zwitterions $\mathbf{1}(2.40)$ and $\mathbf{2}(1.74)$ (Figure 6 and Table S4). The large energy difference between the two orbitals makes the molecule to prefer the closed-shell configuration. However, small energy gap between the two orbitals makes them energetically degenerate and hence, open shell configuration can be easily achieved by promoting an electron from HOMO into the LUMO, leading to the preference of open-shell configuration in the molecule. SOMOs play a significant role in the 
magnetic interaction owing to itinerant exchange. It is already reported that low SOMOSOMO gap or nearly degenerate SOMOs foster strong magnetic exchange interactions. ${ }^{64,82,83}$ From Figure 6b, it has been observed that the energy difference between the two SOMOs i.e. $\Delta \mathrm{E}_{S S}$ is smaller for diradicals $\mathbf{3}, \mathbf{4}, \mathbf{5}$ and $\mathbf{6}$ in comparison with the larger value for the zwitterion 1 and $\mathbf{2}$. Consequently, the nearly degenerate SOMOs prefer open shell configurations in comparison to the closed-shell state for non-degenerate SOMOs.

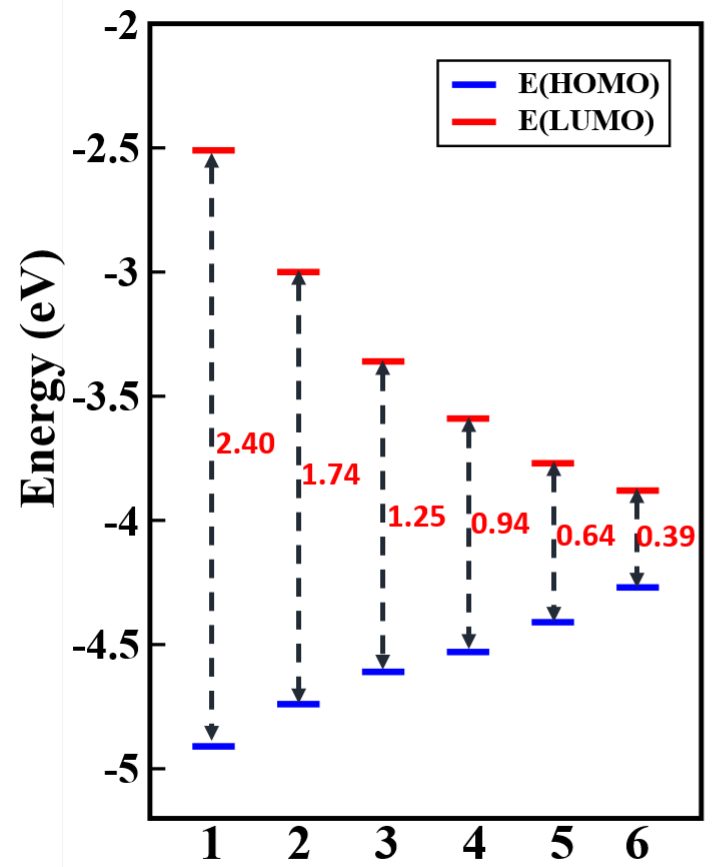

(a.)

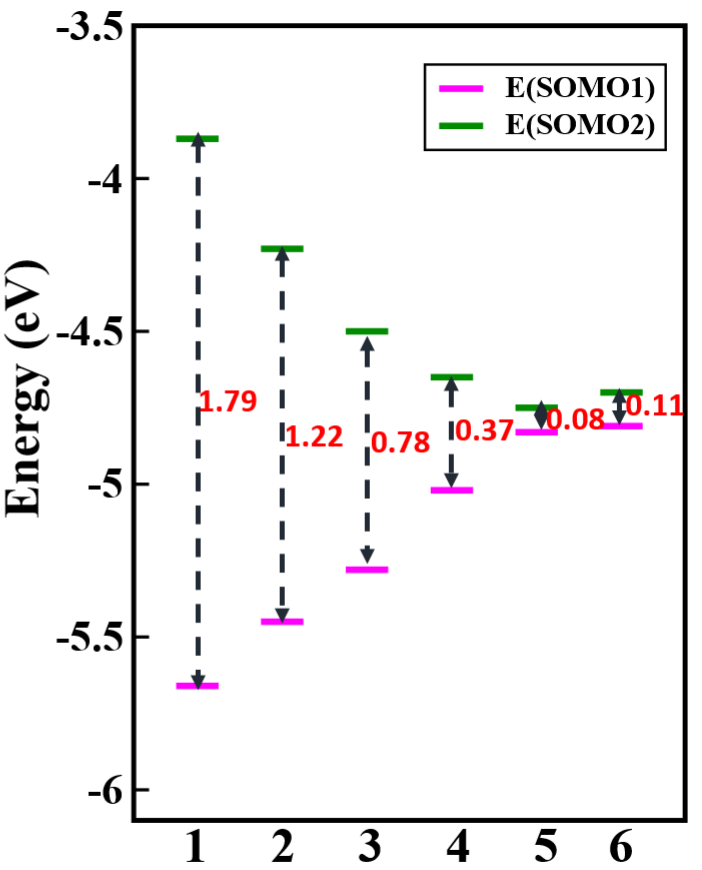

(b.)

Figure 6: Energy difference (eV) between (a) HOMO and LUMO in the closed shell singlet state. (b) SOMO1 and SOMO2 in the triplet state. The blue and red lines represent HOMO and LUMO respectively and pink and green lines represent SOMO1 and SOMO2 respectively.

The computed magnetic exchange interactions for the diradicals 3, 4, 5 and 6 from different methods i.e. BS-DFT and wavefunction based CASSCF and CASSCF-NEVPT2 are collected in Figure 7 and Table 1, S5 and S6. The traditional BS-DFT employing the hybrid B3LYP functional results in intramolecular antiferromagnetic exchange interactions for diradicals 3 and $\mathbf{4}$ whereas diradicals $\mathbf{5}$ and $\mathbf{6}$ are characterized by ferromagnetic spinspin coupling characteristics. A switching from open-shell singlet to triplet configuration is 
observed while moving from molecule 4 to 5 . Since, DFT functionals tend to produce overestimated magnitude of couplings due to exceeding spin-delocalization in the highly conjugated systems, ${ }^{84,85}$ it becomes mandatory to employ symmetry-adapted wave function theory based multiconfigurational methods i.e. CASSCF and CASSCF-NEVPT2 to compute the exchange interactions in these diradicals. We have selected $\operatorname{CASSCF}(10,10)$ active space, incorporating 10 active electrons in 10 active orbitals, for the estimation of the exchange interactions and the dynamical correlations are incorporated employing NEVPT2. It is observed that for all the diradicals, CASSCF $(10,10)$ and $\operatorname{CASSCF}(10,10)-N E V P T 2$ results in similar characteristics of exchange interactions i.e. antiferromagnetic for diradical $\mathbf{3}$ and $\mathbf{4}$ and ferromagnetic coupling for $\mathbf{5}$ and $\mathbf{6}$. Although the sign of exchange interactions is consistently reproduced by the wave-function based methods, the expected lowering in the magnitude of antiferromagnetic and ferromagnetic exchange coupling is observed from WFT based methods than that obtained from BS-DFT for all the diradicals. The CASSCF/NEVPT2 incorporating static as well as dynamical correlations provides huge antiferromagnetic exchange coupling constant with value of $-1551.44 \mathrm{~cm}^{-1}$ for diradical 3 and a moderate value of -447.03 and $37.10 \mathrm{~cm}^{-1}$ for diradical 4 and 5 respectively with antiferromagnetic and ferromagnetic exchange. A giant intramolecular ferromagnetic exchange interactions with $2 J$ of $950.10 \mathrm{~cm}^{-1}$ is calculated for diradical $\mathbf{6}$ making it the best possible candidate for organic molecular magnets. 


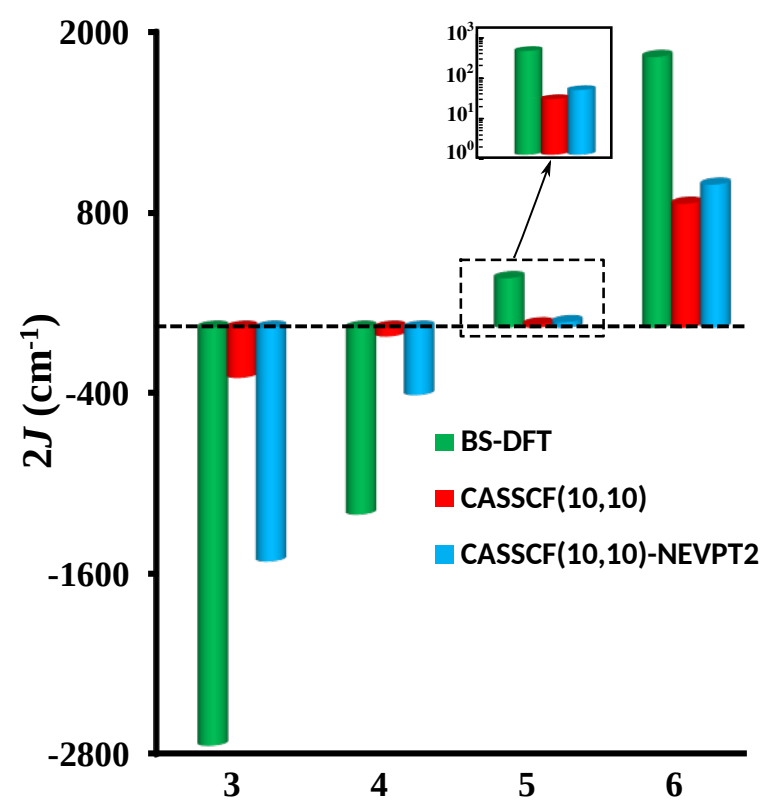

Figure 7: Computed magnetic exchange interactions obtained with DFT and wavefunction based CASSCF and CASSCF/NEVPT2 methods for the molecules $\mathbf{3}, \mathbf{4}, \mathbf{5}$ and $\mathbf{6}$. Molecules 3 and $\mathbf{4}$ are stablized in open-shell singlet ground state with antiferromagnetic interactions and $\mathbf{5}$ and $\mathbf{6}$ exhibits ferromagnetic exchange with triplet ground state.

For the in depth understanding of the nature of exchange interactions in these molecules, the prerequisite is to understand the nature of coupler between the two spin centers. A conventional example of coupler that manifests ferromagnetic interactions is the m-phenylene coupler that is suitable for both carbon-centered as well as nitrogen-centered radicals such as nitroxyl and nitronyl nitroxide (NN) radicals. ${ }^{27,86}$ Although in some cases, it is observed as an antiferromagnetic coupler. In that case, it depends on the conformation of the spin sources with respect to the coupler. ${ }^{87,88}$ It has been observed that when the dihedral angle between the spin sources and coupler is large enough, it forbids the conjugation between the radical and coupler. Hence, the well known nature of ferromagnetic coupling of coupler is overturned. Tetraphenylhexaazaanthracene (TPHA), i.e. molecule $\mathbf{1}$ also contains the m-phenylene coupler and is expected to be a ferromagnetic diradical. However, to overcome its potential antiaromaticity, it exists in the zwitterionic configuration with two electrons occupying the same orbital and is observed to be diamagnetic in nature with large HOMOLUMO gap. However, as the number of benzene rings between the two radical centers i.e. the 
distance between the two radical centers increases, energy gap between HOMO and LUMO decreases. It leads to the starting of the phenomenon of bond dissociation and as a result, the bond distance between the two electrons starts increasing making the two electrons to exist in the isolated environment. Consequently, it results in the preference of open-shell singlet configurations for molecule 3 and $\mathbf{4}$. With further increasing the number of benzene rings, bond dissociation limit is achieved which instigates the intrinsic behavior of m-phenylene coupler to give ferromagnetic interactions between the two radical centers. Hence, for $\mathbf{5}$ and $\mathbf{6}$, ferromagnetic coupling is observed with very small energy gap between HOMO and LUMO. This transformation from closed shell to open shell configuration is well correlated to the dissociation of bond in a simple homodinuclear molecule by Nakano. ${ }^{89}$ The strategy of enhancing the diradical character by increasing the spacing between the radical centers has been successfully applied by Rottschafer et al. They have observed a decrease in the singlet-triplet energy gap with increasing the spacer between the radical centers. ${ }^{90}$

\subsection{Substituent effect}

In order to investigate the simultaneous effect of different electron withdrawing (EWG) and electron donating groups (EDG) i.e. push-pull substitution on the ground-spin state of TPHA, we investigated a series of model systems (shown in Figure 8) sharing a common skeleton of TPHA with the substitution of hydrogens of the central benzene ring with different EWG and EDG. With the aim of designing molecules with larger diradical character and hence diradicaloid or triplet ground state, a large variety of substituents with wide range of inductive and resonating effects are employed. The designing strategy is to simultaneously push the electrons by substituting EDG (in place of $\mathrm{H}$ ) in the already electron rich negative part and to pull the electrons by substituting EWG from the already electron deficient positive part of the molecule (Figure 8). 


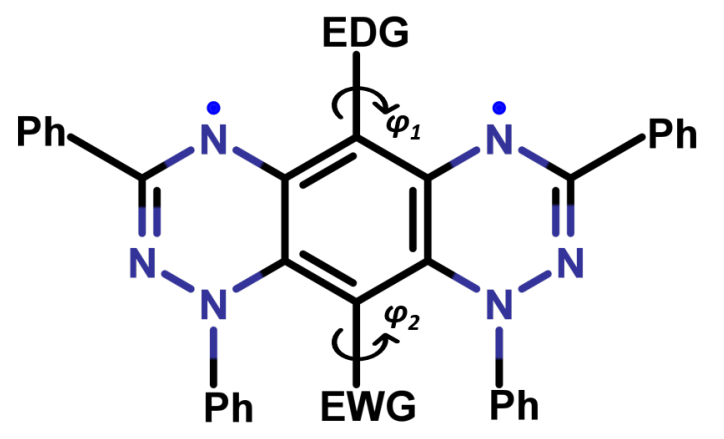

$\begin{array}{ccc}\text { Molecule } & \text { EDG } & \text { EWG } \\ \mathbf{1} & \mathrm{H} & \mathrm{H} \\ \mathbf{1 a} & \mathrm{NMe}_{2} & \mathrm{CF}_{3} \\ \mathbf{1 b} & \mathrm{NMe}_{2} & \mathrm{CN} \\ \mathbf{1 c} & \mathrm{NMe}_{2} & \mathrm{NO}_{2} \\ \mathbf{1 d} & \mathrm{NH}_{2} & \mathrm{CF}_{3} \\ \mathbf{1 e} & \mathrm{NH}_{2} & \mathrm{CN} \\ \mathbf{1 f} & \mathrm{NH}_{2} & \mathrm{NO}_{2} \\ \mathbf{1 g} & \mathrm{OH} & \mathrm{CF}_{3} \\ \mathbf{1 h} & \mathrm{OH} & \mathrm{CN}^{-} \\ \mathbf{1 i} & \mathrm{OH} & \mathrm{NO}_{2}\end{array}$

Figure 8: Modeled diradicals designed with the simultaneous substitution of electron donating group (EDG) and electron withdrawing group (EWG) on the parent TPHA i.e. molecule $\mathbf{1}$ and are aliased as $\mathbf{1 a}$ to $\mathbf{1 i}$.

The electron-donating substituents employed are $\mathrm{NMe}_{2}, \mathrm{NH}_{2}$ and $\mathrm{OH}$ and electronwithdrawing substituents are $\mathrm{CF}_{3}, \mathrm{CN}$ and $\mathrm{NO}_{2}$. The substituents $\mathrm{NMe}_{2}, \mathrm{NH}_{2}$ and $\mathrm{OH}$ have positive resonating effect $(+\mathrm{R})$ implying electron-donating nature whereas $\mathrm{CN}$ and $\mathrm{NO}_{2}$ possess negative resonating effect (-R) leading to electron-withdrawing nature and $\mathrm{CF}_{3}$ owing to negative inductive effect (-I) also shows electron-withdrawing character. Further, the strength of donation or removal of electron density of different substituents is provided by Hammett constant $\left(\sigma_{\text {para }}\right)^{91}$ which signifies the net influence of resonating and inductive effects. The negative and positive sign of $\sigma_{\text {para }}$ signifies electron-donating and electronwithdrawing nature of substituent respectively and its magnitude represents the strength of donation or removal of electron density. From the Hammett constants provided in Table S7, it is observed that $\mathrm{NMe}_{2}, \mathrm{NH}_{2}$ and $\mathrm{OH}$ have negative $\sigma_{\text {para }}$ implying electron-donating character with the strength in the order $\mathrm{NMe}_{2}(-0.83)>\mathrm{NH}_{2}(-0.66)>\mathrm{OH}(-0.37)$ whereas $\mathrm{NO}_{2}$, $\mathrm{CN}$ and $\mathrm{CF}_{3}$ possess positive value indicating electron-withdrawing nature of substituents with $\mathrm{NO}_{2}(0.78)$ being strongest followed by $\mathrm{CN}(0.66)$ and $\mathrm{CF}_{3}(0.54)$.

Further, based on attached EDG, the 9 modeled molecules (shown in Figure 8) are classified into three distinct series $\mathrm{A}, \mathrm{B}$ and $\mathrm{C}$ containing $\mathrm{NMe}_{2}, \mathrm{NH}_{2}$ and $\mathrm{OH}$ respectively as EDG. EWGs are varied from $\mathrm{CF}_{3}, \mathrm{CN}$ and $\mathrm{NO}_{2}$ in each series respectively. Accordingly, molecules $\mathbf{1 a}, \mathbf{1 b}$ and $\mathbf{1 c}$ belongs to series A, series $\mathrm{B}$ contains $\mathbf{1 d}$, $\mathbf{1 e}$ and $\mathbf{1 f}$, and series $\mathrm{C}$ 
comprises of $\mathbf{1 g}, \mathbf{1 h}$ and $\mathbf{1 i}$.

The investigation of $<S^{2}>$ values in HS state (Table 2) reveals that the spin-contaminations of the triplet states are low and are atmost 0.04 . However, the $<S^{2}>$ values in BS state show wide range of spin-contamination with value of $<S^{2}>$ ranging from 0.23 to 0.62 . Since, the $<\mathrm{S}^{2}>_{B S}$ values render the extent of diradical character wherein the large $<\mathrm{S}^{2}>_{B S}$ values approaching towards $1.0(<1.0)$ signifies the diradicaloid ground state of the molecules. Appreciably, all the substituted systems are found to possess larger $<\mathrm{S}^{2}>_{B S}$ values than the parent molecule $\mathbf{1}$. To rationalise the observed increase in diradical character upon substitution, we propose that the EDG pushes its electrons to the central fused benzene ring and simultaneously the EWG pulls the electrons from the benzene ring resulting in a pushpull mechanism leading to a resonating structure $\mathbf{Y}$ (Figure 9). We believe that with this resonating structure, the molecule will no longer be able to partition its $16 \pi$ electrons into $10 \pi$-negative and $6 \pi$-positive parts and thus displays a transition from zwitterionic TPHA to a diradicaloid state. Apart from this, the calculated HOMA value of the central benzene ring (Table 2) is in the range of $0.47-0.61$, indicating lesser $\pi$-orbital overlap within the ring. Although the aromaticity of the central ring is still sacrificed which can be quantified from the smaller HOMA values, but the loss of aromaticity of the ring is not due to partitioning into negative and positive components but it is because of existence of resonance in these molecules as structures $\mathbf{X}$ and $\mathbf{Y}($ Figure 9). Due to these resonating structures, the two unpaired electrons will be localized on the radical centers (triazinyl rings) instead of taking part in the partitioning into two components. Eventually, the increase in the molecular diradical character and the diradicaloid ground state will be encountered.

Further, in all the three series, the molecule containing $\mathrm{NO}_{2}$ as EWG i.e. 1c, $\mathbf{1 f}$ and 1i displays larger $<\mathrm{S}^{2}>_{B S}$ among their respective series and thus possess larger diradical character. This is due to the strongest electron-removing tendency of the $\mathrm{NO}_{2}$ substituent among the three EWGs considered as quantified by its Hammett constant. Among these molecules, 1c comes as an optimum combination that provides the largest value which is 

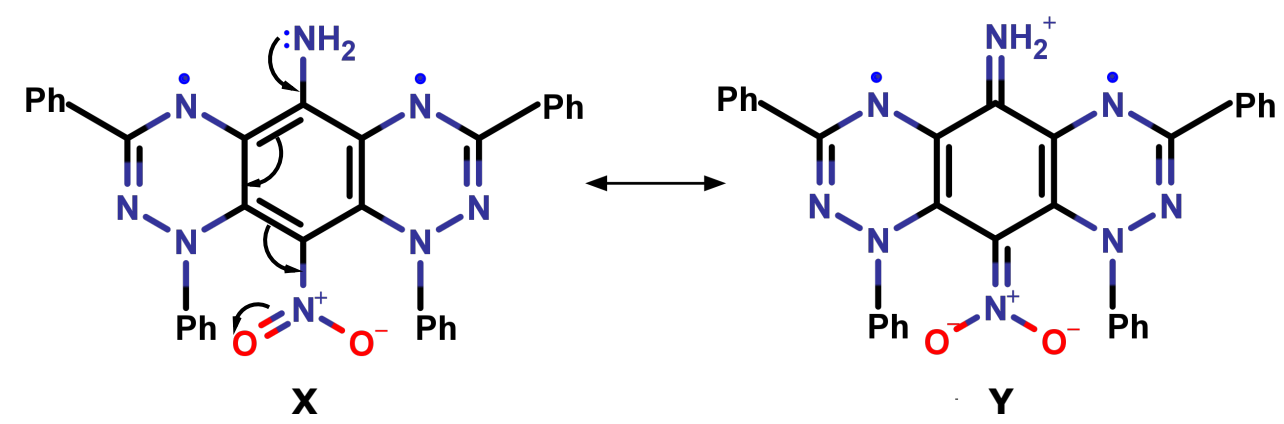

Figure 9: The resonance in the substituted molecules with the resonating structures $\mathbf{X}$ and $\mathbf{Y}$. Here, resonating structures of molecule $\mathbf{1 c}$ is shown.

due to strongest electron-donating ability of $\mathrm{NMe}_{2}$ followed by $\mathrm{NH}_{2}$ and $\mathrm{OH}$ which is in line to their electron-donation strength. However, molecules $\mathbf{1 b}, \mathbf{1 e}$ and $\mathbf{1 h}$ containing $\mathrm{CN}$ as EWG yields larger value with $\mathrm{NH}_{2}$ followed by $\mathrm{NMe}_{2}$ as EDG which is in contrast to the electron donating strength of the substituent. The source of this ambiguity is found in the interplanar angles $\left(\phi_{1}\right.$ and $\phi_{2}$ shown in Figure 8) which the EDG and EWG makes with the benzene ring (Table S8). It has been observed that in 1e, the EDG is perfectly planar to the benzene ring yielding interplanar angle $\sim 120^{\circ}$ whereas $\mathbf{1 b}$ shows a minute deviation of $1.5^{\circ}$ from the plane leading to slightly less overlap of $2 \mathrm{p}$ orbitals of $\mathrm{N}$ atom of EDG and $\mathrm{C}$ atom of benzene ring as compared to 1 e where the perfect planarity yields appropriate overlapping of the orbitals. It leads to facile donation of lone pair of nitrogen of EDG to the benzene ring through resonance and hence, makes $\mathbf{Y}$ the dominant resonating structure. Thus, the source of mismatch of the electron-donating nature of substituent can be attributed to the electronic structure of the molecules. The same argument also applies to explain the result observed with $\mathrm{CF}_{3}$ as EDG where $\mathrm{NH}_{2}(\mathbf{1 d})$ shows larger value than $\mathrm{NMe}_{2}(\mathbf{1 a})$ due to ideally planar angle in $\mathbf{1 d}$ than $\mathbf{1 a}$ with slight distortion of $\sim 1.5^{\circ}$. 
Table 2: The $\left\langle S^{2}\right\rangle$ values in HS and BS state for the molecules 1a-1i obtained with different substituents and the HOMA values of the central benzene ring of all the molecules.

\begin{tabular}{cccccc}
\hline Molecule & EDG & EWG & $\left\langle S^{2}\right\rangle_{H S}$ & $\left\langle S^{2}\right\rangle_{B S}$ & HOMA \\
\hline $\mathbf{1}$ & $\mathrm{H}$ & $\mathrm{H}$ & 2.03 & 0.23 & 0.58 \\
\hline $\mathbf{1 a}$ & $\mathrm{NMe}_{2}$ & $\mathrm{CF}_{3}$ & 2.04 & 0.49 & 0.48 \\
$\mathbf{1 b}$ & $\mathrm{NMe}_{2}$ & $\mathrm{CN}$ & 2.04 & 0.48 & 0.49 \\
$\mathbf{1 c}$ & $\mathrm{NMe}_{2}$ & $\mathrm{NO}_{2}$ & 2.04 & 0.62 & 0.47 \\
\hline $\mathbf{1 d}$ & $\mathrm{NH}_{2}$ & $\mathrm{CF}_{3}$ & 2.04 & 0.57 & 0.61 \\
$\mathbf{1 e}$ & $\mathrm{NH}_{2}$ & $\mathrm{CN}$ & 2.04 & 0.58 & 0.57 \\
$\mathbf{1 f}$ & $\mathrm{NH}_{2}$ & $\mathrm{NO}_{2}$ & 2.04 & 0.58 & 0.62 \\
\hline $\mathbf{1 g}$ & $\mathrm{OH}$ & $\mathrm{CF}_{3}$ & 2.04 & 0.46 & 0.56 \\
$\mathbf{1 h}$ & $\mathrm{OH}$ & $\mathrm{CN}$ & 2.04 & 0.39 & 0.64 \\
$\mathbf{1 i}$ & $\mathrm{OH}$ & $\mathrm{NO}_{2}$ & 2.04 & 0.47 & 0.57 \\
\hline
\end{tabular}

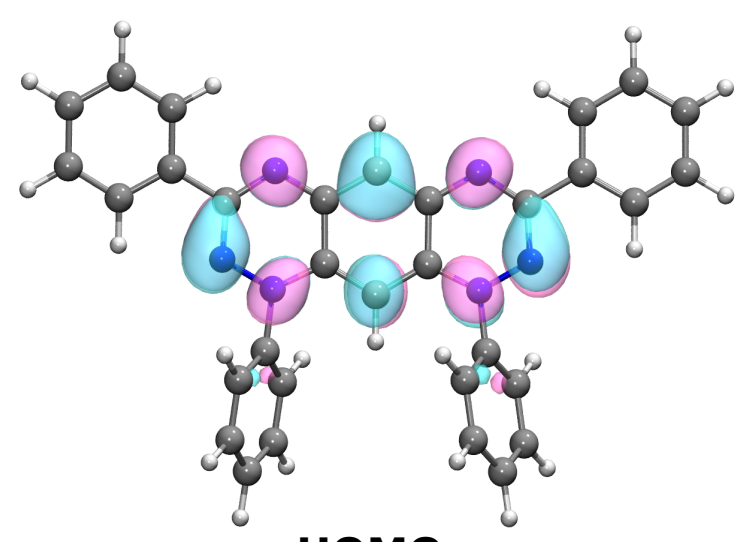

HOMO

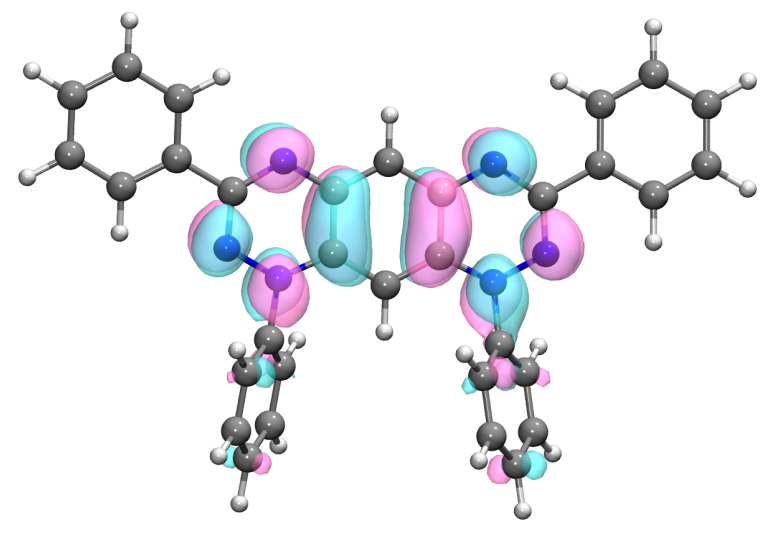

LUMO

Figure 10: Molecular orbitals (HOMO and LUMO) of molecule 1 i.e. TPHA in the closedshell singlet state at B3LYP/def2-TZVP level at an isovalue of 0.03.

Further, the inspection of the frontier molecular orbitals of TPHA reveals that HOMO of TPHA exhibits large atomic coefficient at the centre of $10 \pi$-anionic part and LUMO shows a node at the centre of $6 \pi$-cationic part (Figure 10). Similar spatial features of frontier orbitals were also observed previously for bis(1,2,3-dithiazoles). ${ }^{63}$ From this peculiar distribution of frontier orbitals, one can infer that the introduction of EDG will donate electrons to the electron rich position and will raise the energy level of HOMO. Similarly, introduction of EWG at positive position will remove electrons from the electron deficient position and will lower the energy level of LUMO. Therefore, the simultaneous substitution of EDG and 
EWG results in the raising of HOMO level and lowering of LUMO level and consequently, brings down the gap between HOMO and LUMO $\left(\Delta \mathrm{E}_{H L}\right)$ leading to preferential diradicaloid ground state of the molecules. Figure 11 depicts the $\Delta \mathrm{E}_{H L}$ of the molecules with different EDG and EWG and the parent molecule 1. It has been identified that for $\mathbf{1}$, the $\Delta \mathrm{E}_{H L}$ is highest with least diradical character and for all other molecules, $\Delta \mathrm{E}_{H L}$ is smaller than that for $\mathbf{1}$. This proposes an inverse relation between the $\Delta \mathrm{E}_{H L}$ and the diradical character. Among the three series, $\Delta \mathrm{E}_{H L}$ is least for the molecules containing $\mathrm{NO}_{2}$ as EWG i.e. 1c, 1f and 1i providing the reason for the large diradical character in these molecules. The smallest $\Delta \mathrm{E}_{H L}$ for molecule $1 \mathbf{c}$ also unveils the observation of largest diradical character in this molecule. In series $\mathrm{A}$, molecules $\mathbf{1 a}$ and $\mathbf{1} \mathbf{b}$ bear similar $\Delta \mathrm{E}_{H L}$ supporting comparable diradical character. In series $\mathrm{B}$, molecules $1 \mathbf{d}$ and $1 \mathbf{e}$ possess equivalent $\Delta \mathrm{E}_{H L}$ matching the corresonding diradical character. Among series $\mathrm{C}$, molecule $\mathbf{1 h}$ with smallest diradical character bears largest $\Delta \mathrm{E}_{H L}$.

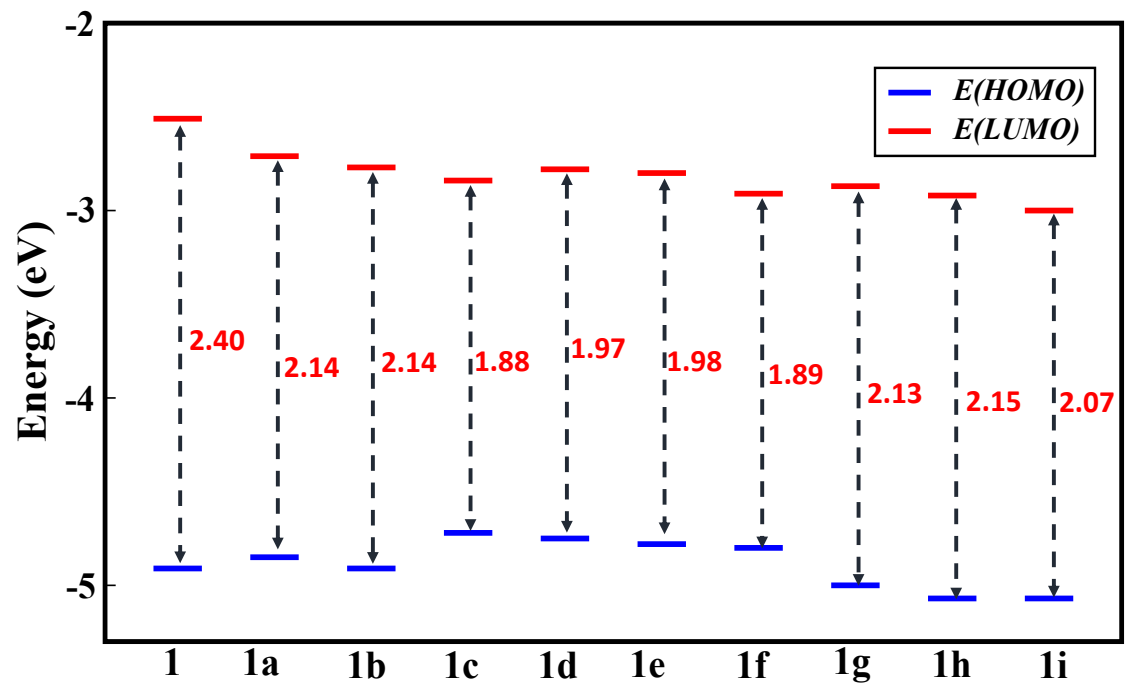

Figure 11: Energy difference $(\mathrm{eV})$ between HOMO and LUMO in the structures optimized in open-shell singlet state for the molecules with different EDG and EWG.

Furthermore, while exploring the effect of different push-pull substitutions on the diradical character, we encountered three molecules (Figure S2) which exhibit pure diradical ground state. These are designed by positioning $\mathrm{O}^{-}$as EDG and $\mathrm{CF}_{3}, \mathrm{CN}$ and $\mathrm{NO}_{2}$ as EWG 
and acronymed as $\mathrm{O}-\mathrm{CF}_{3}, \mathrm{O}-\mathrm{CN}$ and $\mathrm{O}-\mathrm{NO}_{2}$ respectively. For all the three diradicals, the $<S^{2}>_{B S}$ values are $\sim 1.0$ (Table S9) manifesting ideal diradical character in these molecules. Moreover, the LUNO occupation number from $\operatorname{CASSCF}(2,2)$ i.e. $\mathrm{n}_{L U N O}$ is $\sim 0.70$ (Table S10) signifying $70 \%$ diradical character in all the molecules. The HOMA values calculated for the central benzene ring of these diradicals is $\sim 0.20$ (Table S11) which is much smaller than those of molecules $\mathbf{1 a - 1 i}$ revealing the loss of aromaticity of the ring is much more pronounced in these diradicals. This imparts that the resonating structure $\mathbf{Y}$ (Figure 9) is more significant than $\mathbf{X}$ which is also observed from the $\mathrm{C}-\mathrm{O}$ bond length in the three radicals which acquires double bond character with bond length of $1.23 \AA$. The magnetic exchange interactions are calculated from BS-DFT as well as WFT based CASSCF $(2,2)$ and CASSCF(2,2)-NEVPT2 (Table S9 and S12). From all the aforementioned methods, strong ferromagnetic exchange interactions are calculated for all the three diradicals owing to triplet ground state as illustrated in Figure 12.

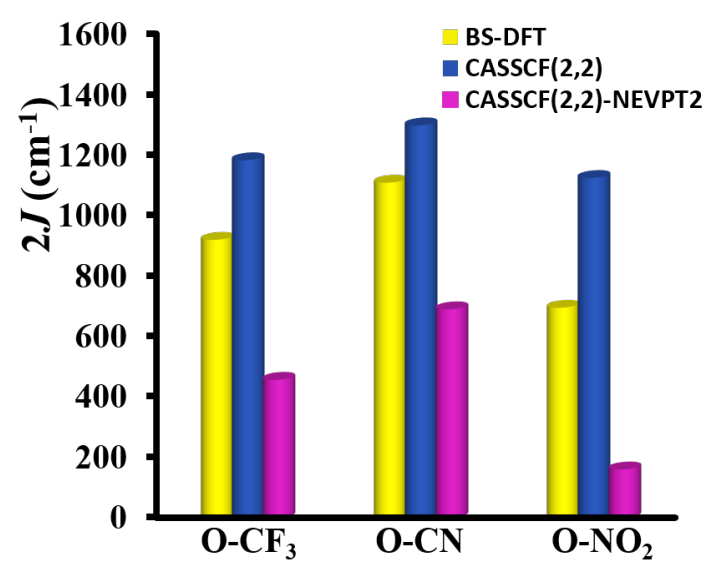

Figure 12: Computed magnetic exchange interactions in three diradicals i.e. $\mathrm{O}-\mathrm{CF}_{3}, \mathrm{O}-\mathrm{CN}$ and $\mathrm{O}-\mathrm{NO}_{2}$.

Thus, the increase of diradical character and in consequence, attainment of diradicaloid or triplet ground state, upon simultaneous substitution of EDG and EWG comes up as a beneficial implementation for the designing of molecules with diradicalized ground state advancing a step closer towards superior magnetic materials. 


\section{Conclusion}

With the aim of instigating diradical character in the well known zwitterionic ground-state of tetraphenylhexaazaanthracene i.e. TPHA (here, molecule 1), the molecule encompassing two Blatter's radical moieties, two distinct strategies have been employed. In the first approach, the length of the coupler between the two radical centers is increased upto hexaacene. It has been identified that with increasing the length of the coupler, the diradical character in a molecule is increased. A transition from closed-shell singlet (molecule $\mathbf{1}$ and $\mathbf{2})$ to open-shell singlet (3 and $\mathbf{4}$ ) and subsequently to triplet state (5 and $\mathbf{6}$ ) is observed with the enlargement of length of the coupler. For assessment of diradical character, two different indexes, DFT based spin squared-value $\left(<\mathrm{S}^{2}>_{B S}\right)$ and WFT based LUNO occupation number $\left(\mathrm{n}_{L U N O}\right)$, are implemented which complements well with each other. Both of them reveals increase in diradical character with increase in the number of benzene rings. Further, strong intramolecular ferromagnetic exchange coupling is reported for molecule 6 proposing it as a potential organic molecular magnet. Moderate ferromagnetic coupling is also observed for molecule 5. A good correlation is observed between magnetic exchange coupling constants and the energy gap between the closed-shell singlet and triplet state.

Another alternative approach is the substitution of electron donating group (EDG) and electron withdrawing group (EWG) simultaneously (push-pull substitution) at the anionic and cationic fragments of the zwitterionic molecule 1. It has been discovered that this strategic substitution on molecules with zwitterionic ground-state proposes a plausible way to alter the diradical character of the molecule leading to the diradicaloid ground-state. Furthermore, three molecules are observed which exhibits strong intramolecular ferromagnetic

exchange interactions owing to triplet ground state. This work provides a new perspective of designing molecules based on Blatter's radical with ferromagnetic ground state. 


\section{Acknowledgement}

R.K. thanks CSIR, India for the JRF fellowship with grant number 09/1129(0016)/2019EMR-I. Financial support from Department of Science and Technology through SERB-ECR project No. ECR/2016/000362 and SERB-CRG project No. CRG/2019/003237 are highly acknowledged.

Supporting Information Available: Energetics of different molecules, computed exchange couplings using DFT and WFT based methods, diradicals with ferromagnetic coupling.

\section{References}

(1) Gopalakrishna, T. Y.; Zeng, W.; Lu, X.; Wu, J. From open-shell singlet diradicaloids to polyradicaloids. Chem. Commun. 2018, 54, 2186-2199.

(2) Morita, Y.; Suzuki, S.; Sato, K.; Takui, T. Synthetic organic spin chemistry for structurally well-defined open-shell graphene fragments. Nat. Chem. 2011, 3, 197.

(3) Sanvito, S. Molecular spintronics. Chem. Soc. Rev. 2011, 40, 3336-3355.

(4) Morita, Y.; Nishida, S.; Murata, T.; Moriguchi, M.; Ueda, A.; Satoh, M.; Arifuku, K.; Sato, K.; Takui, T. Organic tailored batteries materials using stable open-shell molecules with degenerate frontier orbitals. Nat. Mater. 2011, 10, 947-951.

(5) Kubo, T.; Shimizu, A.; Sakamoto, M.; Uruichi, M.; Yakushi, K.; Nakano, M.; Shiomi, D.; Sato, K.; Takui, T.; Morita, Y., et al. Synthesis, intermolecular interaction, and semiconductive behavior of a delocalized singlet biradical hydrocarbon. Angew. Chem. Int. Ed. 2005, 44, 6564-6568.

(6) Hu, X.; Wang, W.; Wang, D.; Zheng, Y. The electronic applications of stable diradicaloids: present and future. J. Mater. Chem. C 2018, 6, 11232-11242. 
(7) Rajca, A. Organic diradicals and polyradicals: from spin coupling to magnetism? Chem. Rev. 1994, 94, 871-893.

(8) Sun, Z.; Ye, Q.; Chi, C.; Wu, J. Low band gap polycyclic hydrocarbons: from closedshell near infrared dyes and semiconductors to open-shell radicals. Chem. Soc. Rev. 2012, 41, 7857-7889.

(9) Abe, M.; Ye, J.; Mishima, M. The chemistry of localized singlet 1, 3-diradicals (biradicals): from putative intermediates to persistent species and unusual molecules with a $\pi$-single bonded character. Chem. Soc. Rev. 2012, 41, 3808-3820.

(10) Abe, M. Diradicals. Chem. Rev. 2013, 113, 7011-7088.

(11) Zeng, Z.; Shi, X.; Chi, C.; Navarrete, J. T. L.; Casado, J.; Wu, J. Pro-aromatic and anti-aromatic $\pi$-conjugated molecules: an irresistible wish to be diradicals. Chem. Soc. Rev. 2015, 44, 6578-6596.

(12) Ni, Y.; Wu, J. Diradical approach toward organic near infrared dyes. Tetrahedron Lett. 2016, 57, 5426-5434.

(13) Tan, G.; Wang, X. Isolable bis (triarylamine) dications: analogues of Thiele's, Chichibabin's, and Muller's hydrocarbons. Acc. Chem. Res. 2017, 50, 1997-2006.

(14) Ovchinnikov, A. A. Multiplicity of the ground state of large alternant organic molecules with conjugated bonds. Theor. Chim. Acc. 1978, 47, 297-304.

(15) Thiele, J.; Balhorn, H. Ueber einen chinoïden Kohlenwasserstoff. Ber. Dtsch. Chem. Ges. 1904, 3\%, 1463-1470.

(16) Tschitschibabin, A. Über einige phenylierte Derivate des p, p-Ditolyls. Ber. Dtsch. Chem. Ges. 1907, 40, 1810-1819.

(17) Kubo, T.; Shimizu, A.; Sakamoto, M.; Uruichi, M.; Yakushi, K.; Nakano, M.; Shiomi, D.; Sato, K.; Takui, T.; Morita, Y., et al. Synthesis, intermolecular interaction, 
and semiconductive behavior of a delocalized singlet biradical hydrocarbon. Angew. Chem. Int. Ed. 2005, 44, 6564-6568.

(18) Shimizu, A.; Kubo, T.; Uruichi, M.; Yakushi, K.; Nakano, M.; Shiomi, D.; Sato, K.; Takui, T.; Hirao, Y.; Matsumoto, K., et al. Alternating covalent bonding interactions in a one-dimensional chain of a phenalenyl-based singlet biradical molecule having Kekulé structures. J. Am. Chem. Soc. 2010, 132, 14421-14428.

(19) Casado, J. Physical Organic Chemistry of Quinodimethanes; Springer, 2017; pp 209248.

(20) Rudebusch, G. E.; Zafra, J. L.; Jorner, K.; Fukuda, K.; Marshall, J. L.; ArrecheaMarcos, I.; Espejo, G. L.; Ortiz, R. P.; Gómez-García, C. J.; Zakharov, L. N., et al. Diindeno-fusion of an anthracene as a design strategy for stable organic biradicals. Nat. Chem. 2016, 8, 753-759.

(21) Frederickson, C. K.; Rose, B. D.; Haley, M. M. Explorations of the indenofluorenes and expanded quinoidal analogues. Acc. Chem. Res. 2017, 50, 977-987.

(22) Zeng, W.; Sun, Z.; Herng, T. S.; Gonçalves, T. P.; Gopalakrishna, T. Y.; Huang, K.-W.; Ding, J.; Wu, J. Super-heptazethrene. Angew. Chem. Int. Ed. 2016, 55, 8615-8619.

(23) Konishi, A.; Hirao, Y.; Kurata, H.; Kubo, T.; Nakano, M.; Kamada, K. Anthenes: Model systems for understanding the edge state of graphene nanoribbons. Pure Appl. Chem. 2014, 86, 497-505.

(24) Ali, M. E.; Datta, S. N. Broken-symmetry density functional theory investigation on bis-nitronyl nitroxide diradicals: Influence of length and aromaticity of couplers. $J$. Phys. Chem. A 2006, 110, 2776-2784.

(25) Tretyakov, E. V.; Zhivetyeva, S. I.; Petunin, P. V.; Gorbunov, D. E.; Gritsan, N. P.; Bagryanskaya, I. Y.; Bogomyakov, A. S.; Postnikov, P. S.; Kazantsev, M. S.; 
Trusova, M. E., et al. Ferromagnetically coupled $\mathrm{S}=1$ chains in crystals of verdazylnitronyl nitroxide diradicals. Angew. Chem. Int. Ed. 2020, 59, 20704-20710.

(26) Latif, I. A.; Panda, A.; Datta, S. N. Very strongly ferromagnetically coupled diradicals from mixed radical centers: nitronyl nitroxide coupled to oxoverdazyl via polyene spacers. J. Phys. Chem. A 2009, 113, 1595-1600.

(27) Gallagher, N. M.; Olankitwanit, A.; Rajca, A. High-spin organic molecules. J. Org. Chem. 2015, 80, 1291-1298.

(28) Fedyushin, P.; Rybalova, T.; Asanbaeva, N.; Bagryanskaya, E.; Dmitriev, A.; Gritsan, N.; Kazantsev, M.; Tretyakov, E. Synthesis of nitroxide diradical using a new approach. Molecules 2020, 25, 2701.

(29) Ali, M. E.; Datta, S. N. Density functional theory prediction of enhanced photomagnetic properties of nitronyl nitroxide and imino nitroxide diradicals with substituded dihydropyrene couplers. J. Phys. Chem. A 2006, 110, 10525-10527.

(30) Ali, M. E.; Vyas, S.; Datta, S. N. Ab initio quantum chemical investigation of intramolecular magnetic interaction in some diradical derivatives of imino nitroxide and nitronyl nitroxide. J. Phys. Chem. A 2005, 109, 6272-6278.

(31) Dressler, J. J.; Valdivia, A. C.; Kishi, R.; Rudebusch, G. E.; Ventura, A. M.; Chastain, B. E.; Gómez-García, C. J.; Zakharov, L. N.; Nakano, M.; Casado, J., et al. Diindenoanthracene diradicaloids enable rational, incremental tuning of their singlettriplet energy gaps. Chem 2020, 6, 1353-1368.

(32) Vyas, S.; Ali, M. E.; Hossain, E.; Patwardhan, S.; Datta, S. N. Theoretical investigation of intramolecular magnetic interaction through an ethylenic coupler. J. Phys. Chem. A 2005, 109, 4213-4215. 
(33) Chen, Z.; Li, Y.; Huang, F. Persistent and stable organic radicals: design, synthesis, and applications. Chem 2021, 7, 288-332.

(34) Blatter, H. M.; Lukaszewski, H. A new stable free radical. Tetrahedron Lett. 1968, 9, $2701-2705$.

(35) Constantinides, C. P.; Koutentis, P. A.; Loizou, G. Synthesis of 7-aryl/heteraryl-1, 3-diphenyl-1, 2, 4-benzotriazinyls via palladium catalyzed Stille and Suzuki-Miyaura reactions. Org. Biomol. Chem. 2011, 9, 3122-3125.

(36) Constantinides, C. P.; Koutentis, P. A.; Krassos, H.; Rawson, J. M.; Tasiopoulos, A. J. Characterization and magnetic properties of a "Super Stable" radical 1, 3-Diphenyl-7trifluoromethyl-1, 4-dihydro-1, 2, 4-benzotriazin-4-yl. J. Org. Chem. 2011, 76, 27982806.

(37) Kaszyński, P.; Constantinides, C. P.; Young Jr, V. G. The planar blatter radical: structural chemistry of 1, 4-Dihydrobenzo [e][1, 2, 4] triazin-4-yls. Angew. Chem. 2016, 128, 11315-11318.

(38) Rogers, F.; Norcott, P.; Coote, M. Recent advances in the chemistry of Benzo [e][1, 2, 4] triazinyl radicals. Org. Biomol. Chem. 2020, 18, 8255-8277.

(39) Ji, Y.; Long, L.; Zheng, Y. Recent advances of stable Blatter radicals: synthesis, properties and applications. Mater. Chem. Front. 2020, 4, 3433-3443.

(40) Kumar, S.; Kumar, Y.; Keshri, S. K.; Mukhopadhyay, P. Recent advances in organic radicals and their magnetism. Magnetochemistry 2016, 2, 42.

(41) Takahashi, Y.; Matsuhashi, R.; Miura, Y.; Yoshioka, N. Magnetic interactions through a nonconjugated framework observed in back-to-back connected triazinyl-nitroxyl biradical derivatives. Chem. Eur. J. 2018, 24, 7939-7948. 
(42) Karecla, G.; Papagiorgis, P.; Panagi, N.; Zissimou, G. A.; Constantinides, C. P.; Koutentis, P. A.; Itskos, G.; Hayes, S. C. Emission from the stable Blatter radical. New J. Chem. 2017, 41, 8604-8613.

(43) Ciccullo, F.; Gallagher, N.; Geladari, O.; Chassé, T.; Rajca, A.; Casu, M. A derivative of the Blatter radical as a potential metal-free magnet for stable thin films and interfaces. ACS Appl. Mater. Interfaces 2016, 8, 1805-1812.

(44) Sidharth, T. N. S.; Nasani, R.; Gupta, A.; Sooraj, B. N. S.; Roy, S.; Mondal, A.; Konar, S. Reversal of magnetic exchange coupling between copper (II) and Blatter radical depending on the coordination environment. Inorganica Chim. Acta 2020, 503, 119395.

(45) Gallagher, N. M.; Bauer, J. J.; Pink, M.; Rajca, S.; Rajca, A. High-spin organic diradical with robust stability. J. Am. Chem. Soc. 2016, 138, 9377-9380.

(46) Gallagher, N.; Zhang, H.; Junghoefer, T.; Giangrisostomi, E.; Ovsyannikov, R.; Pink, M.; Rajca, S.; Casu, M. B.; Rajca, A. Thermally and magnetically robust triplet ground state diradical. J. Am. Chem. Soc. 2019, 141, 4764-4774.

(47) Bajaj, A.; Ali, M. E. First-principle design of Blatter's diradicals with strong ferromagnetic exchange interactions. J. Phys. Chem. C 2019, 123, 15186-15194.

(48) Bajaj, A.; Khurana, R.; Ali, M. E. Auxiliary atomic relay center facilitates enhanced magnetic couplings in Blatter's radical. 2021, DOI: 10.26434/chemrxiv.13513881.v1.

(49) Zhang, Y.; Zheng, Y.; Zhou, H.; Miao, M.-S.; Wudl, F.; Nguyen, T.-Q. Temperature Tunable Self-Doping in Stable Diradicaloid Thin-Film Devices. Adv. Mater. 2015, 27, $7412-7419$.

(50) Hu, X.; Chen, H.; Zhao, L.; Miao, M.-s.; Zheng, X.; Zheng, Y. Nitrogen-coupled blatter diradicals: the fused versus unfused bridges. J. Mater. Chem. C 2019, 7, 10460-10464. 
(51) Hu, X.; Chen, H.; Zhao, L.; Miao, M.; Han, J.; Wang, J.; Guo, J.; Hu, Y.; Zheng, Y. Nitrogen analogues of Chichibabin's and Müller's hydrocarbons with small singlet-triplet energy gaps. Chem. Commun. 2019, 55, 7812-7815.

(52) Hu, X.; Chen, H.; Xue, G.; Zheng, Y. Correlation between the strength of conjugation and spin-spin interactions in stable diradicaloids. J. Mater. Chem. C 2020, 8, 1074910754.

(53) Khurana, R.; Bajaj, A.; Ali, M. E. How plausible is getting ferromagnetic interactions by coupling Blatter's radical via its fused benzene ring? J. Phys. Chem. A 2020, 124, $6707-6713$.

(54) Zissimou, G. A.; Berezin, A. A.; Manoli, M.; Nicolaides, C.; Trypiniotis, T.; Koutentis, P. A. 3, 3', 3"-(Benzene-1, 3, 5-triyl) tris (1-phenyl-1H-benzo [e][1, 2, 4] triazin-4-yl): A C3 symmetrical Blatter-type triradical. Tetrahedron 2020, 76, 131077.

(55) Hutchison, K.; Srdanov, G.; Hicks, R.; Yu, H.; Wudl, F.; Strassner, T.; Nendel, M.; Houk, K. Tetraphenylhexaazaanthracene: A Case for Dominance of Cyanine Ion Stabi-

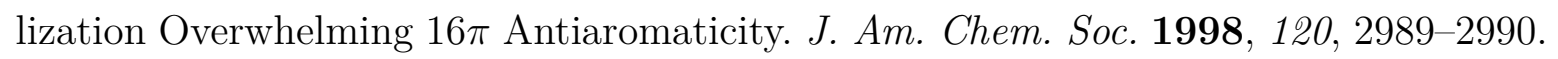

(56) Langer, P.; Amiri, S.; Bodtke, A.; Saleh, N. N.; Weisz, K.; Gorls, H.; Schreiner, P. R. 3, 5, 7, 9-Substituted Hexaazaacridines: Toward Structures with Nearly Degenerate Singlet- Triplet Energy Separations. J. Org. Chem. 2008, 73, 5048-5063.

(57) Constantinides, C. P.; Zissimou, G. A.; Berezin, A. A.; Ioannou, T. A.; Manoli, M.; Tsokkou, D.; Theodorou, E.; Hayes, S. C.; Koutentis, P. A. Tetraphenylhexaazaanthracenes: $16 \pi$ Weakly Antiaromatic Species with Singlet Ground States. Org. Lett. 2015, 17, 4026-4029.

(58) Haas, Y.; Zilberg, S. Charge separation in ground-state 1, 2, 4, 5-tetra-substituted benzene derivatives. J. Am. Chem. Soc. 2004, 126, 8991-8998. 
(59) Langer, P.; Bodtke, A.; Saleh, N. N.; Görls, H.; Schreiner, P. R. 3, 5, 7, 9Tetraphenylhexaazaacridine: A Highly Stable, Weakly Antiaromatic Species with $16 \pi$ Electrons. Angew. Chem. Int. Ed. 2005, 44, 5255-5259.

(60) Braunstein, P.; Siri, O.; Taquet, J.-p.; Rohmer, M.-M.; Bénard, M.; Welter, R. A $6 \pi+$ $6 \pi$ potentially antiaromatic zwitterion preferred to a quinoidal structure: Its reactivity toward organic and inorganic reagents. J. Am. Chem. Soc. 2003, 125, 12246-12256.

(61) Bendikov, M.; Duong, H. M.; Starkey, K.; Houk, K.; Carter, E. A.; Wudl, F. Oligoacenes: theoretical prediction of open-shell singlet diradical ground states. J. Am. Chem. Soc. 2004, 126, 7416-7417.

(62) Hachmann, J.; Dorando, J. J.; Avilés, M.; Chan, G. K.-L. The radical character of the acenes: a density matrix renormalization group study. J. Chem. Phys. 2007, 127, 134309.

(63) Constantinides, C. P.; Ioannou, T. A.; Koutentis, P. A. Manipulating the singlet-triplet energy gaps of arene-fused bis (1, 2, 3-dithiazoles): A computational study. Polyhedron 2013, 64, 172-180.

(64) Zhang, G.; Li, S.; Jiang, Y. Effects of Substitution on the Singlet- Triplet Energy Splittings and Ground-State Multiplicities of m-Phenylene-Based Diradicals: A Density Functional Theory Study. J. Phys. Chem. A 2003, 10\%, 5573-5582.

(65) Guevara-Level, P.; Pascal, S.; Siri, O.; Jacquemin, D. First principles investigation of the spectral properties of neutral, zwitterionic, and bis-cationic azaacenes. Phys. Chem. Chem. Phys. 2019, 21, 22910-22918.

(66) Noodleman, L. Valence bond description of antiferromagnetic coupling in transition metal dimers. J. Chem. Phys. 1981, 74, 5737-5743. 
(67) Kruszewski, J.; Krygowski, T. Definition of aromaticity basing on the harmonic oscillator model. Tetrahedron Lett. 1972, 13, 3839 - 3842.

(68) Krygowski, T. M.; Cyrański, M. Separation of the energetic and geometric contributions to the aromaticity. Part IV. A general model for the $\pi$-electron systems. Tetrahedron 1996, 52, $10255-10264$.

(69) Becke, A. D. A new mixing of Hartree-Fock and local density-functional theories. J. Chem. Phys. 1993, 98, 1372-1377.

(70) Lee, C.; Yang, W.; Parr, R. G. Development of the Colle-Salvetti correlation-energy formula into a functional of the electron density. Phys. Rev. B 1988, 37, 785-789.

(71) Weigend, F.; Ahlrichs, R. Balanced basis sets of split valence, triple zeta valence and quadruple zeta valence quality for $\mathrm{H}$ to $\mathrm{Rn}$ : Design and assessment of accuracy. Phys. Chem. Chem. Phys. 2005, 7, 3297-3305.

(72) Neese, F.; Wennmohs, F.; Hansen, A.; Becker, U. Efficient, approximate and parallel Hartree-Fock and hybrid DFT calculations. A 'chain-of-spheres' algorithm for the Hartree-Fock exchange. Chem. Phys. 2009, 98-109.

(73) Shil, S.; Herrmann, C. Performance of range-separated hybrid exchange-correlation functionals for the calculation of magnetic exchange coupling constants of organic diradicals. J. Comput. Chem. 2018, 39, 780-787.

(74) Illas, F.; Moreira, I. P.; De Graaf, C.; Barone, V. Magnetic coupling in biradicals, binuclear complexes and wide-gap insulators: a survey of ab initio wave function and density functional theory approaches. Theor. Chem. Acc. 2000, 104, 265-272.

(75) Reta Maneru, D.; Pal, A. K.; Moreira, I. d. P.; Datta, S. N.; Illas, F. The Triplet-Singlet Gap in the m-Xylylene Radical: A Not So Simple One. J. Chem. Theory Comput. 2014, $10,335-345$. 
(76) Malrieu, J. P.; Caballol, R.; Calzado, C. J.; de Graaf, C.; Guihery, N. Magnetic interactions in molecules and highly correlated materials: physical content, analytical derivation, and rigorous extraction of magnetic Hamiltonians. Chem. Rev. 2014, 114, 429-492.

(77) Khedkar, A.; Roemelt, M. Active space selection based on natural orbital occupation numbers from n-electron valence perturbation theory. J. Chem. Theory Comput. 2019, $15,3522-3536$.

(78) Angeli, C.; Cimiraglia, R.; Evangelisti, S.; Leininger, T.; Malrieu, J.-P. Introduction of n-electron valence states for multireference perturbation theory. J. Chem. Phys. 2001, $114,10252-10264$.

(79) Neese, F. The ORCA program system. Wiley Interdiscip. Rev. Comput. Mol. Sci. 2012, 2, $73-78$.

(80) Kaur, P.; Ali, M. E. First Principle Investigations of Long-range Magnetic Exchange Interactions via Polyacene Coupler. 2020, DOI: 10.26434/chemrxiv.12776222.v1.

(81) Yang, H.; Chen, M.; Song, X.; Bu, Y. Structural fluctuation governed dynamic diradical character in pentacene. Phys. Chem. Chem. Phys. 2015, 17, 13904-13914.

(82) Hoffmann, R.; Zeiss, G. D.; Van Dine, G. W. The electronic structure of methylenes. J. Am. Chem. Soc. 1968, 90, 1485-1499.

(83) Constantinides, C. P.; Koutentis, P. A.; Schatz, J. A DFT study of the ground state multiplicities of linear vs angular polyheteroacenes. J. Am. Chem. Soc. 2004, 126, $16232-16241$.

(84) David, G.; Guihéry, N.; Ferré, N. What Are the Physical Contents of Hubbard and Heisenberg Hamiltonian Interactions Extracted from Broken Symmetry DFT Calculations in Magnetic Compounds? J. Chem. Theory Comput. 2017, 13, 6253-6265. 
(85) Grau-Crespo, R.; Corà, F.; Sokol, A. A.; de Leeuw, N. H.; Catlow, C. R. A. Electronic structure and magnetic coupling in $\mathrm{FeSbO}_{4}$ : A DFT study using hybrid functionals and GGA + U methods. Phys. Rev. B 2006, 73, 035116.

(86) Pal, A. K.; Mañeru, D. R.; Latif, I. A.; de PR Moreira, I.; Illas, F.; Datta, S. N. Theoretical and computational investigation of meta-phenylene as ferromagnetic coupler in nitronyl nitroxide diradicals. Theor. Chem. Acc. 2014, 133, 1-12.

(87) Fang, S.; Lee, M.-S.; Hrovat, D. A.; Borden, W. T. Ab initio calculations show why m-phenylene is not always a ferromagnetic coupler. J. Am. Chem. Soc. 1995, 117, $6727-6731$.

(88) Zhang, J.; Zhang, H.; Wang, L.; Wang, R.; Wang, L. Effect of configuration and conformation on the spin multiplicity in xylylene type biradicals. Sci. China, Ser. B: Chem. $2000,43,524-530$.

(89) Nakano, M. Electronic structure of open-shell singlet molecules: Diradical character viewpoint. Top. Curr. Chem. 2017, 375, 47.

(90) Rottschäfer, D.; Busch, J.; Neumann, B.; Stammler, H.-G.; Van Gastel, M.; Kishi, R.; Nakano, M.; Ghadwal, R. S. Diradical Character Enhancement by Spacing: NHeterocyclic Carbene Analogues of Müller's Hydrocarbon. Chem. Eur. J. 2018, 24, 16537-16542.

(91) Hammett, L. P. The effect of structure upon the reactions of organic compounds. Benzene derivatives. J. Am. Chem. Soc. 1937, 59, 96-103. 